\begin{tabular}{ccc}
\hline Jij & Journal of Mathematical Analysis and Modeling & J Math Anal \& Model \\
ISmam.sabapub.com & $(2020) 1(1): 20-32$ \\
ISSN 2709-5924 & doi:10.48185/jmam.v1i1.23 \\
\hline
\end{tabular}

\title{
Approximate fixed points for $n$-Linear functional by $(\mu, \sigma)$ - nonexpansive Mappings on $n$-Banach spaces
}

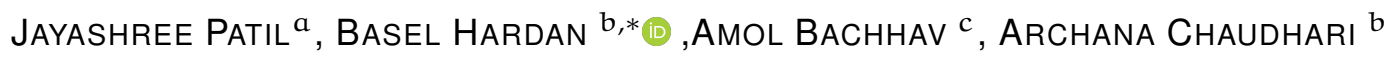 \\ a Department of Mathematics, Vasantrao Naik Mahavidyalaya, Cidco, Aurangabad (M.S.), \\ India. \\ ${ }^{b}$ Department of Mathematics, Dr. Babasaheb Ambedkar Marathwada University, \\ Aurangabad, India. \\ ${ }^{c}$ School of Management, University of Texas at Dallas, Dallas, 75080.
}

• Received: 07.10.2020 • Accepted/Published Online: 18.11.2020 • Final Version: 03.12.2020

\begin{abstract}
In this paper, we conclude that $n$-linear functionals spaces $\mathfrak{I}$ has approximate fixed points set, where $\mathfrak{I}$ is a non-empty bounded subset of an $n$-Banach space $\mathrm{H}$ under the condition of equivalence, and we also use class of $(\mu, \sigma)$-nonexpansive mappings.

Keywords: $\mathrm{n}$-linear functional, $\mathrm{n}$-normed spaces, $\mathrm{n}$-inner product spaces, $\mathrm{n}$-Banach spaces, $(\mu, \sigma)$-nonexpansive mapping, Fixed point set. 2010 MSC: 34A08, 34B15, 34A12, 47H10.
\end{abstract}

\section{Introduction}

Functional analysis studies the spaces of functions and the processes operated on them, among which are the normed space and the inner product spaces. They are the basis for studying many concepts in the different branches of mathematics and measuring the vector and non-vector quantities in physics. Thus, when studying these spaces with equal dimensions or greater than the $n^{\text {th }}$ dimensions, we get to measure the sizes between $n$ vectors that make up the different spaces. In this case, it leads to generalizing and rereading many terms for the various branches of science that study these two spaces. Thus, the importance of this research lies here. The idea of $n$-normed spaces was first presented by Gahler [1, 2, 3]. The idea of $n$-inner product spaces was created by Misiak [4]. As we realize that the length of a vector is a subject we talk about on normed spaces, in n-normed spaces we talk about the volume of a parallelepiped which is crossed by a set comprised of $n$-vectors. There are some results, though they are few that addressed these spaces in their study. For example, Batkunde et al., introduced the topological properties

*Corresponding author: bassil2003@gmail.com

(c) 2020 SABA. All Rights Reserved. 
with respect to its quotient space of $n$-normed spaces, see $([5,6,7])$. Basic properties, fuzzy cases and $n$-distance of $n$-normed spaces were found in $([8,9,10])$. Sequences on n-normed spaces were discussed by Ekariani et al. in [11, 12, 13]. Also, fixed point theory on bounded set was introduced by Meiti et. al., in [14] and Gunawan et. al., in [15]. On the other hand, the theory of fixed point started by Banach in[16] by contraction mapping and was then developed and circulated by many researchers from that time until now. They obtained their results using contraction, contractive or nonexpansive mapping. In the current years, few speculations of nonexpansive mapings have gotten consideration and their fixed point theorems have been concentrated by numerous creators, (see[17, $18,19,20,21,22,23,24,25,26])$. Researchers are thus looking into the possibility of a solution and its uniqueness to different forms of spaces under changing conditions. From these spaces, we chose in our work n-linear functional space on $n$-normed and n-inner product space. Few types of researches study the existence and uniqueness of the solution on n-normed and n-inner product spaces in such cases. If the researcher can prove his results with the addition of a complete $n$-normed spaces we will get results on $n$-Banach spaces, recent studies such as these are mentioned in [27]. In this paper, we will prove that $n$-linear functions have the existence and uniqueness set of the solution on $n$-normed, $n$-inner product spaces using Xianbing's results in [20] by type of $(\mu, \sigma)$-non-expansion mappings.

\section{Preliminaries}

We will recall the following Preliminary facts so as to plan for our results.

Definition 2.1. Let $\mathrm{H}$ be a real linear space $\operatorname{dim} \mathrm{H} \geqslant n$, and let $\|\cdot, \cdot\|: H \times H \rightarrow \mathbb{R}$ such that the following are hold

(i) $\left\|h_{1}, \ldots, h_{n}\right\|=0$ if and only if, $h_{1}, \ldots, h_{n}$ are linearly dependent for all $h_{1}, \ldots, h_{n} \in H$;

(ii) $\left\|h_{1}, \ldots, h_{n}\right\|=\left\|h_{i_{1}}, \ldots, h_{i_{n}}\right\|$, for all $\left(i_{1}, \ldots, i_{n}\right) \in(1, \ldots, n)$;

(iii) $\left\|k h_{1}, \ldots, h_{n}\right\|=|k| \mid h_{1}, \ldots, h_{n} \|, k \in \mathbb{R}$;

(iv) $\left\|h_{1}+\grave{h}_{1}, h_{2}, \ldots, h_{n}\right\| \leqslant\left\|h_{1}, \ldots, h_{n}\right\|+\left\|\grave{h}_{1}, \ldots h_{n}\right\|$, for all $h_{1}, \grave{h_{1}}, \ldots, h_{n} \in H$. Then $\|\cdot, \cdot\|$ is an $n$-normed on $H$ and $(H,\|\cdot, \ldots, \cdot\|)$ is a linear $n$-normed space. We can see that $\left\|h_{1}, \ldots, h_{n}\right\| \geqslant 0$.

In the same direction, Batkund et al.[28] add

$$
\left\|h_{1}+k_{2} h_{2}+\cdots+k_{n} h_{n}, h_{2}, \ldots, h_{n}\right\|=\left\|h_{1}, h_{2}, \ldots, h_{n}\right\|,
$$

for all $h_{1}, h_{2}, \ldots, h_{n} \in H$ and $k_{2}, \ldots, k_{n} \in \mathbb{R}$

Definition 2.2. Let $H$ be a real linear space $\operatorname{dim} H \geqslant n+1$. A function $\langle\cdot, \cdot \mid \cdot, \ldots, \cdot\rangle$ : $\mathrm{H} \times \mathrm{H} \rightarrow \mathbb{R}$ such that the following are hold

(i) $\left\langle h_{1}, h_{1} \mid h_{2}, \ldots, h_{n}\right\rangle \geqslant 0$ and $\left\langle h_{1}, h_{1} \mid h_{2}, \ldots, h_{n}\right\rangle=0$ if and only if $h_{1}, \ldots, h_{n}$ are linearly dependent; 
(ii) $\left\langle h_{1}, h_{1} \mid h_{2}, \ldots, h_{n}\right\rangle=\left\langle h_{i_{1}}, h_{i_{1}} \mid h_{i_{2}}, \ldots, h_{i_{n}}\right\rangle$, for all $\left(i_{1}, \ldots, i_{n}\right) \in(1, \ldots, n)$;

(iii) $\left\langle\grave{h}_{1}, h_{1} \mid h_{2}, \ldots, h_{n}\right\rangle=\left\langle h_{1}, \grave{h}_{1} \mid h_{2}, \ldots, h_{n}\right\rangle$;

(iv) $\left\langle k h_{1}, h_{1} \mid h_{2}, \ldots, h_{n}\right\rangle=|k|\left\langle h_{1}, h_{1} \mid h_{2}, \ldots, h_{n}\right\rangle, k \in \mathbb{R}$;

(v) $\left\langle h_{0}+\grave{h}_{0}, h_{1} \mid h_{2}, \ldots, h_{n}\right\rangle=\left\langle h_{0}, h_{1} \mid h_{2}, \ldots, h_{n}\right\rangle+\left\langle\grave{h}_{0}, h_{1} \mid h_{2}, \ldots, h_{n}\right\rangle$, for all $h_{0}, \grave{h}_{0}, h_{1}, \ldots, h_{n} \in H$.

The pair $(H,\langle\cdot, \cdot \mid \cdot, \ldots, \cdot\rangle)$ is called an $n$-inner product spaces. The relation between $n$-normed spaces and $n$-inner product spaces was referenced by Kristi et al. [29], as $\left\|h_{1}, h_{2}, \ldots, h_{n}\right\|^{2}=\left\langle h_{1}, h_{1} \mid h_{2}, \ldots, h_{n}\right\rangle$.

Example 2.3. If $(H,\|\cdot, \ldots, \cdot\|)$ is an $n$-normed spaces and $H^{\prime}$ it is containing of bounded $\mathrm{n}$-linear functionals on $\mathrm{H}$, we can define the following function on $\mathrm{H}$

$$
\left\|h_{1}, \ldots, h_{n}\right\|^{G}:=\sup _{g_{i} \in H^{\prime},\left\|g_{1}, \ldots, g_{n}\right\| \leqslant 1}\left|\begin{array}{ccc}
g_{1}\left(h_{1}\right) & \cdots & g_{1}\left(h_{n}\right) \\
\vdots & \ddots & \vdots \\
g_{1}\left(h_{n}\right) & \cdots & g_{n}\left(h_{n}\right)
\end{array}\right| .
$$

Example 2.4. If $(H,\langle\cdot, \cdot \mid \ldots, \cdot\rangle)$ is an $n$-Inner product spaces, we can characterize the standard n-norm on $\mathrm{H}$ as

$$
\left\|h_{1}, \ldots, h_{n}\right\|^{S}:=\left|\begin{array}{ccc}
\left\langle h_{1}, h_{1}\right\rangle & \cdots & \left\langle h_{1}, h_{n}\right\rangle \\
\vdots & \ddots & \vdots \\
\left\langle h_{n}, h_{1}\right\rangle & \cdots & \left\langle h_{n}, h_{n}\right\rangle
\end{array}\right|^{\frac{1}{2}} .
$$

Determinate (2.2) is known to always a non-negative Gram's determinate. We rely on changing parameters $g_{i}$ to prevent the negative value from being obtained, while the value of $\left\|h_{1}, \ldots, h_{n}\right\|^{S}$ form the size of parallel from the dimensions of $h_{1}, \ldots, h_{n}$. For more clarification see[30]. In next section, we characterize the class of $(\mu, \sigma)$-non-expansive mappings on $n$-Banach and prove that if $\mathfrak{I}$ be a non-empty bounded form an $n$-Banach spaces, then every $(\mu, \sigma)$-non-expansive mappings $\rho: \mathfrak{I} \rightarrow \mathfrak{I}$ with $\mu>0$ and $\sigma \geqslant 0$ has an approximate fixed point sets.

\section{Main Results}

In this section, we will introduce definitions and thoughts regarding $n$-linear functional on $n$-normed spaces in a different way.

Definition 3.1. Let $(H,\|\cdot, \ldots, \cdot\|)$ be an $n$-normed spaces and $h_{1}, h_{2}, \ldots, h_{n}$ be $n$-subspaces of $H$ and let $\zeta\left(h_{1}, h_{2}, \ldots, h_{n}\right) \subset \mathfrak{I}$. Such that $\mathfrak{I}: \zeta\left(h_{1}, h_{2}, \ldots, h_{n}\right) \rightarrow \mathbb{R}$, then

A function $\zeta\left(h_{1}, h_{2}, \ldots, h_{n}\right)=\left\|h_{1}, \alpha_{i_{1}}, \ldots \alpha_{i_{n}}\right\|\left\|h_{2}, \beta_{i_{1}}, \ldots \beta_{i_{n}}\right\| \ldots\left\|h_{n}, \gamma_{i_{1}}, \ldots \gamma_{i_{n}}\right\|$ is called $n$-linear functinal on $H$ for all $h_{1}, h_{2}, \ldots, h_{n} \in H$ and for all $\left(i_{2}, \cdots, i_{n}\right) \in(1, \cdots, n)$ with $\left(i_{2} \leqslant \cdots \leqslant i_{n}\right)$ and $i_{1} \in\{1, \ldots, n\} \backslash\left\{i_{2}, \ldots, i_{n}\right\}$.

Definition 3.2. A sequence $\zeta\left(h_{k_{1}}\right) \in \mathfrak{I}$ in an $n$-normed space $H$ is said to be converges to some $h_{1} \in H$ if

$$
\lim _{k_{1} \rightarrow \infty}\left\|\zeta\left(h_{k_{1}}\right)-h_{1}, h_{2}, \ldots, h_{n}\right\|=0, \text { for all } h_{1}, \ldots, h_{n} \in H \text { and } k_{1} \in \mathbb{N} \text {. }
$$


Definition 3.3. A sequence $\zeta\left(h_{k_{1}}\right) \in \mathfrak{I}$ in an $n$-normed space $H$ is said to be Cauchy if

$$
\lim _{k_{1}, k_{2} \rightarrow \infty}\left\|\zeta\left(h_{k_{1}}\right)-\zeta\left(h_{k_{2}}\right)_{1}, h_{2}, \ldots, h_{n}\right\|=0, \text { for all } h_{1}, \ldots, h_{n} \in H \text { and } k_{1}, k_{2} \in \mathbb{N} \text {. }
$$

Corollary 3.4. Every convergence sequence in $\mathfrak{I}$ on $\mathrm{H}$ is Cauchy sequence.

Definition 3.5. A function $\zeta\left(h_{1}, h_{2}, \ldots, h_{n}\right) \subset \mathfrak{I} \rightarrow \mathbb{R}$ is satisfies an $n$-Lipschtz condition if there is $\tau \geqslant 1$ such that

$$
\left\|\zeta h_{1}-\zeta h, \zeta h_{2}-\zeta h, \ldots, \zeta h_{n}-\zeta h\right\| \leqslant \tau\left\|h_{1}-h, h_{2}-h, \ldots, h_{n}-h\right\|,
$$

for all $h, h_{1}, h_{2}, \ldots, h_{n} \in H, \tau$ is Called $n$-Lipscitz constant.

Definition 3.6. Let $\left(H_{1},\|\cdot, \ldots, \cdot\|\right)_{h_{1}},\left(H_{2},\|\cdot, \ldots, \cdot\|\right)_{h_{2}}, \ldots,\left(H_{n},\|\cdot, \ldots, \cdot\|\right)_{h_{n}}$ be the $n$-normed spaces and let $\alpha=\left\{\alpha_{i_{1}}, \ldots, \alpha_{i_{n}}\right\}_{\alpha}, \beta=\left\{\beta_{i_{1}}, \ldots, \beta_{i_{n}}\right\}_{\beta}, \ldots, \gamma=\left\{\gamma_{i_{1}}, \ldots, \gamma_{i_{n}}\right\}_{\gamma}$ be a fix linearly independent sets. An n-linear functional $\zeta: \mathrm{H}_{1} \times \mathrm{H}_{2} \times \cdots \times \mathrm{H}_{\mathrm{n}} \rightarrow \mathbb{R}$ is said to be bounded of first index with respect to a pairs of $\alpha, \beta, \ldots, \gamma$, if there exist $M \in \mathbb{R}$ such that:

$$
\begin{aligned}
\left|\zeta\left(h_{1}, h_{2}, \ldots, h_{n}\right)\right| \leqslant M & \left(\left\|h_{1}, \alpha_{i_{1}}, \ldots, \alpha_{i_{n}}\right\|_{H_{1}}\right) \times\left(\left\|h_{2}, \beta_{i_{1}}, \ldots, \beta_{i_{n}}\right\|_{H_{2}}\right) \\
& \times \cdots \times\left(\left\|h_{n}, \gamma_{i_{1}}, \ldots, \gamma_{i_{n}}\right\|_{H_{n}}\right),
\end{aligned}
$$

for all $h_{1} \in H_{1}, \ldots h_{n} \in H_{n}$, and $\left(i_{2}, \cdots, i_{n}\right) \in(1, \cdots, n)$ with $\left(i_{2} \leqslant \cdots \leqslant i_{n}\right)$ such that $i_{1} \in\{1, \ldots, n\} \backslash\left\{i_{2}, \ldots, i_{n}\right\}$.

Definition 3.7. Let $\left(H_{1},\|\cdot, \ldots, \cdot\|\right)_{h_{1}},\left(H_{2},\|\cdot, \ldots, \cdot\|\right)_{h_{2}}, \ldots,\left(H_{n},\|\cdot, \ldots, \cdot\|\right)_{h_{n}}$ be the $n$-normed spaces and let $\alpha=\left\{\alpha_{i_{1}}, \ldots, \alpha_{i_{n}}\right\}_{\alpha}, \beta=\left\{\beta_{i_{1}}, \ldots, \beta_{i_{n}}\right\}_{\beta}, \ldots, \gamma=\left\{\gamma_{i_{1}}, \ldots, \gamma_{i_{n}}\right\}_{\gamma}$ be a fix linearly independent sets. An n-linear functional $\zeta: \mathrm{H}_{1} \times \mathrm{H}_{2} \times \cdots \times \mathrm{H}_{\mathrm{n}} \rightarrow \mathbb{R}$ and $1 \leqslant \tau \leqslant \infty$, is said to be bounded of $\tau^{\text {th }}$ index with respect to a pairs of $\alpha, \beta, \ldots, \gamma$, if there exist $M \in \mathbb{R}$ such that:

$$
\begin{aligned}
\left|\zeta\left(h_{1}, h_{2}, \ldots, h_{n}\right)\right| \leqslant M & \left(\left\|h_{1}, \alpha_{i_{1}}, \ldots, \alpha_{i_{n}}\right\|_{H_{1}}^{\tau}\right)^{\frac{1}{\tau}} \times\left(\left\|h_{2}, \beta_{i_{1}}, \ldots, \beta_{i_{n}}\right\|_{H_{2}}^{\tau}\right)^{\frac{1}{\tau}} \\
& \times \cdots \times\left(\left\|h_{n}, \gamma_{i_{1}}, \ldots, \gamma_{i_{n}}\right\|_{H_{n}}^{\tau}\right)^{\frac{1}{\tau}},
\end{aligned}
$$

for all $h_{1} \in H_{1}, h_{2} \in H_{2}$ for all $\left(i_{2}, \cdots, i_{n}\right) \in(1, \cdots, n)$ with $\left(i_{2} \leqslant \cdots \leqslant i_{n}\right)$ and $i_{1} \in$ $\{1, \ldots, n\} \backslash\left\{i_{2}, \ldots, i_{n}\right\}$.

We can re-write definition 3.1 on $n$-inner product spaces as

Definition 3.8. Let $(H,\langle\cdot, \cdot \mid, \ldots, \cdot\rangle)$ be an $n$-inner product spaces and let $\zeta\left(h_{1}, h_{2}, \ldots, h_{n}\right) \subset \mathfrak{I}$, such that $\mathfrak{I}: \zeta\left(h_{1}, h_{2}, \ldots, h_{n}\right) \rightarrow \mathbb{R}$, A function

$$
\begin{aligned}
\left|\zeta\left(h_{1}, h_{2}, \ldots, h_{n}\right)\right|= & \left(\sum\left\langle h_{1}, \alpha_{i_{1}} \mid \alpha_{i_{2}}, \ldots, \alpha_{i_{n}}\right\rangle\right) \times\left(\sum\left\langle h_{2}, \beta_{i_{1}} \mid \beta_{i_{2}}, \ldots, \beta_{i_{n}}\right\rangle\right) \\
& \times \cdots \times\left(\sum\left\langle h_{n}, \gamma_{i_{1}} \mid \gamma_{i_{2}}, \ldots, \gamma_{i_{n}}\right\rangle\right),
\end{aligned}
$$

is called $n$-linear functinal on $H$ for all $h_{1}, h_{2}, \ldots, h_{n} \in H$ and for all $\left(i_{2}, \cdots, i_{n}\right) \in$ $(1, \cdots, n)$ with $\left(i_{2} \leqslant \cdots \leqslant i_{n}\right)$ and $i_{1} \in\{1, \ldots, n\} \backslash\left\{i_{2}, \ldots, i_{n}\right\}$. 
In the next procedure, we will show that the $n$-linear functionals $\mathfrak{I}$ are bounded, equivalent, forming an $n$-Banach spaces.

Lemma 3.9. Let $\mathrm{H}$ be an $\mathrm{n}$-normed spaces and let $\left\{\zeta\left(\mathrm{h}_{\mathrm{k}}\right)\right\},\left\{\varphi\left(\mathrm{s}_{\mathrm{k}}\right)\right\} \subset \mathfrak{I} \in \mathrm{H}$ be two Cauchy sequences in n-linear functional spaces then

(a) $\zeta\left(h_{k}\right)$ is a Cauchy sequence on $\mathbb{R}$.

(b) $\left\|\zeta\left(h_{k}\right)+\varphi\left(s_{k}\right), \alpha_{2}, \ldots, \alpha_{n}\right\|$, is Cauchy sequence in $H$,

(c) If $\left\{\zeta\left(h_{k}\right)\right\} \rightarrow h$ and $\left\{\zeta\left(h_{k}\right)\right\} \rightarrow \hbar$, then $h=\hbar, k \rightarrow \infty$.

Proof. Since $\left\{\zeta\left(h_{k}\right)\right\},\left\{\varphi\left(s_{k_{1}}\right)\right\}$ are two Cauchy sequences in $n$-linear functional spaces, we obtain

$$
\begin{aligned}
\left\|\zeta\left(h_{k}\right), \alpha_{2}, \ldots, \alpha_{n}\right\| & =\left\|\left(\zeta\left(h_{k}\right)-\varphi\left(s_{k_{1}}\right)\right)+\varphi\left(s_{k_{1}}\right), \alpha_{2}, \ldots, \alpha_{n}\right\|, \\
& \leqslant\left\|\left(\zeta\left(h_{k}\right)-\varphi\left(s_{k_{1}}\right)\right), \alpha_{2}, \ldots, \alpha_{n}\right\|+\left\|\varphi\left(s_{k_{1}}\right), \alpha_{2}, \ldots, \alpha_{n}\right\|,
\end{aligned}
$$

so, we have

$$
\begin{aligned}
\left\|\zeta\left(h_{k}\right), \alpha_{2}, \ldots, \alpha_{n}\right\| & -\left\|\varphi\left(s_{k_{1}}\right), \alpha_{2}, \ldots, \alpha_{n}\right\| \\
& \leqslant\left\|\left(\zeta\left(h_{k}\right)-\varphi\left(s_{k_{1}}\right)\right), \alpha_{2}, \ldots, \alpha_{n}\right\|+\left\|\varphi\left(s_{k_{1}}\right), \alpha_{2}, \ldots, \alpha_{n}\right\|,
\end{aligned}
$$

and,

$$
\left.\left\|\varphi\left(s_{k_{1}}\right), \alpha_{2}, \ldots, \alpha_{n}\right\|-\| \zeta\left(h_{k}\right)\right), \alpha_{2}, \ldots, \alpha_{n}\|\leqslant\|\left(\zeta\left(h_{k}\right)-\varphi\left(s_{k_{1}}\right)\right), \alpha_{2}, \ldots, \alpha_{n} \|,
$$

consolidating the above, we make sure that

$$
\left|\left\|\zeta\left(h_{k}\right), \alpha_{2}, \ldots, \alpha_{n}\right\|-\left\|\varphi\left(s_{k_{1}}\right), \alpha_{2}, \ldots, \alpha_{n}\right\|\right| \leqslant\left\|\left(\zeta\left(h_{k}\right)-\varphi\left(s_{k_{1}}\right)\right), \alpha_{2}, \ldots, \alpha_{n}\right\| \rightarrow 0,
$$

as $k, k_{1} \rightarrow \infty$. Hence, $\left\|\zeta\left(h_{k}\right), \alpha_{2}, \ldots, \alpha_{n}\right\|$ is a Cauchy sequence in $\mathbb{R}$, (a) is proven. To prove (b), since $\left\{\zeta\left(h_{k}\right)\right\},\left\{\varphi\left(s_{k}\right)\right\}$ are two Cauchy sequences, then

$$
\lim _{k, k_{1} \rightarrow \infty}\left\|\zeta\left(h_{k}\right)-\zeta\left(h_{k_{1}}\right), \alpha_{2}, \ldots, \alpha_{n}\right\| \rightarrow 0, \quad \text { for all } \alpha_{2}, \ldots, \alpha_{n} \in H,
$$

and,

$$
\lim _{k, k_{1} \rightarrow \infty}\left\|\varphi\left(h_{k}\right)-\varphi\left(h_{k_{1}}\right), \alpha_{2}, \ldots, \alpha_{n}\right\| \rightarrow 0, \quad \text { for all } \alpha_{2}, \ldots, \alpha_{n} \in H,
$$

therefore,

$$
\begin{aligned}
& \left\|\left(\zeta\left(h_{k}\right)+\varphi\left(h_{k}\right)-\left(\zeta\left(h_{k_{1}}\right)+\varphi\left(h_{k_{1}}\right)\right)\right), \alpha_{2}, \ldots, \alpha_{n}\right\| \\
& =\left\|\left(\zeta\left(h_{k}\right)-\zeta\left(h_{k_{1}}\right)+\left(\varphi\left(h_{k}\right)-\varphi\left(h_{k_{1}}\right)\right)\right), \alpha_{2}, \ldots, \alpha_{n}\right\|, \\
& \quad \leqslant \|\left(\zeta\left(h_{k}\right)-\zeta\left(h_{k_{1}}\right), \alpha_{2}, \ldots, \alpha_{n}\|+\|\left(\varphi\left(h_{k}\right)-\varphi\left(h_{k_{1}}\right)\right), \alpha_{2}, \ldots, \alpha_{n} \| \rightarrow 0,\right.
\end{aligned}
$$

as $k, k_{1} \rightarrow \infty$. Now, to prove (c) follow

$$
\begin{aligned}
\left\|h-\hbar, \alpha_{2}, \ldots, \alpha_{n}\right\| & =\left\|h-\hbar+\zeta\left(h_{k}\right)-\zeta\left(h_{k}\right), \alpha_{2}, \ldots, \alpha_{n}\right\|, \\
& \leqslant\left\|\zeta\left(h_{k}\right)-h, \alpha_{2}, \ldots, \alpha_{n}\right\|+\left\|\zeta\left(h_{k}\right)-\hbar, \alpha_{2}, \ldots, \alpha_{n}\right\| \rightarrow 0,
\end{aligned}
$$

as $k \rightarrow \infty$. Since $\operatorname{dim} H \geqslant n$ We have one choice to consider that $h-\hbar$ is linearly dependent with $\alpha_{2}, \ldots, \alpha_{n}$, which is $h-\hbar=0$. Hence, $h=\hbar$. 
Corollary 3.10. $\left\|E_{k} \zeta\left(h_{k}\right), \alpha_{1}, \ldots, \alpha_{n}\right\|$ is Cauchy sequences in $H$, such that $E_{k}$ Cauchy in $\mathbb{R}$.

Corollary 3.11. Every $\mathfrak{I} \in \mathrm{H}$ is an $\mathrm{n}$-Banach space.

Theorem 3.12. The following assumptions are holds

(a) n-linear functional (3.6) is bounded of first index with

$$
\|\zeta\|_{1}=\left\|\alpha_{1}, \ldots, \alpha_{n}\right\|_{H_{1}}\left\|\beta_{1}, \ldots, \beta_{n}\right\|_{H_{2}} \cdots\left\|\gamma_{1}, \ldots, \gamma_{n}\right\|_{H_{n}} ;
$$

(b) n-linear functional is bounded of $\tau^{\text {th }}$ index with

$$
\|\zeta\|_{\tau}=n^{\frac{n}{\sigma}}\left\|\alpha_{1}, \ldots, \alpha_{n}\right\|_{H_{1}}\left\|\beta_{1}, \ldots, \beta_{n}\right\|_{H_{2}}\left\|\gamma_{1}, \ldots, \gamma_{n}\right\|_{H_{n}}
$$

where $\frac{\mathrm{n}}{\tau}+\frac{\mathrm{n}}{\sigma}=\mathrm{n}$;

(c)

$$
\begin{aligned}
\|\zeta\|_{1}=: \sup \left\{\left\|\alpha_{1}, \alpha_{2}, \ldots, \alpha_{n}\right\|\left\|\beta_{1}, \beta_{2}, \ldots, \beta_{n}\right\| \ldots\left\|\gamma_{1}, \gamma_{2}, \ldots, \gamma_{n}\right\|,\right. \\
\mid\left(\sum\left\|h_{1}, \alpha_{i_{2}}, \ldots, \alpha_{i_{n}}\right\|_{H_{1}}\right) \leqslant 1,\left(\sum\left\|h_{2}, \beta_{i_{2}}, \ldots, \beta_{i_{n}}\right\|_{H_{2}}\right) \leqslant 1 \\
\left.\ldots,\left(\sum\left\|h_{n}, \gamma_{i_{2}}, \ldots, \gamma_{i_{n}}\right\|_{H_{n}}\right) \leqslant 1\right\}
\end{aligned}
$$

(d)

$$
\begin{aligned}
\|\zeta\|_{\tau}=: \sup \{ & n^{\frac{n}{\sigma}}\left\|\alpha_{1}, \alpha_{2}, \ldots, \alpha_{n}\right\|\left\|\beta_{1}, \beta_{2}, \ldots, \beta_{n}\right\| \ldots\left\|\gamma_{1}, \gamma_{2}, \ldots, \gamma_{n}\right\|, \\
& \mid\left(\sum\left\|h_{1}, \alpha_{i_{2}}, \ldots, \alpha_{i_{n}}\right\|_{H_{1}}^{\tau}\right) \leqslant 1,\left(\sum\left\|h_{2}, \beta_{i_{2}}, \ldots, \beta_{i_{n}}\right\|_{H_{2}}^{\tau}\right) \leqslant 1 \\
& \left.\ldots,\left(\sum\left\|h_{n}, \gamma_{i_{2}}, \ldots, \gamma_{i_{n}}\right\|_{H_{n}}^{\tau}\right) \leqslant 1\right\}
\end{aligned}
$$

(e)

$$
\|\zeta\|_{1} \equiv\|\zeta\|_{\tau}
$$

Proof. Note that, for all $h_{1} \in \mathrm{H}_{1}, h_{2} \in \mathrm{H}_{2}, \ldots, h_{n} \in \mathrm{H}_{\mathrm{n}}$ we get

$$
\begin{aligned}
& \left|\zeta\left(h_{1}, h_{2}, \ldots, h_{n}\right)\right| \leqslant\left\|\alpha_{i_{1}}, \ldots, \alpha_{i_{n}}\right\|_{H_{1}} \times\left\|\beta_{i_{1}}, \ldots, \beta_{i_{n}}\right\|_{H_{2}} \times \cdots \times\left\|\gamma_{i_{1}}, \ldots, \gamma_{i_{n}}\right\|_{H_{n}} \\
\times & \left(\sum\left\|h_{1}, \alpha_{i_{2}}, \ldots, \alpha_{i_{n}}\right\|_{H_{1}}\right)\left(\sum\left\|h_{2}, \beta_{i_{2}}, \ldots, \beta_{h_{n}}\right\|_{H_{2}}\right) \cdots\left(\sum\left\|h_{n}, \gamma_{i_{2}}, \ldots, \gamma_{i_{n}}\right\|_{H_{n}}\right),
\end{aligned}
$$

by using triangle and Cauchy Schwarz' inequality.

If we take

$$
\begin{gathered}
h_{1}=\left\|\alpha_{i_{2}}, \ldots, \alpha_{i_{n}}\right\| \alpha_{1} \\
h_{2}=\left\|\beta_{i_{2}}, \ldots, \beta_{i_{n}}\right\| \beta_{1} \\
\quad \vdots \\
h_{n}=\left\|\gamma_{i_{2}}, \ldots, \gamma_{i_{n}}\right\| \gamma_{1} .
\end{gathered}
$$


Then

$\left(\sum\left\|h_{1}, \alpha_{i_{2}}, \ldots, \alpha_{i_{n}}\right\|\right)=\left(\sum\left\|h_{2}, \beta_{i_{2}}, \ldots, \beta_{h_{n}}\right\|\right)=, \ldots,=\left(\sum\left\|h_{n}, \gamma_{i_{2}}, \ldots, \gamma_{i_{n}}\right\|\right)=1$, and,

$$
\begin{aligned}
\left|\zeta\left(h_{1}, h_{2}, \ldots, h_{n}\right)\right| & =\left\|\alpha_{1}, \alpha_{2}, \ldots, \alpha_{n}\right\|^{-1}\left\|\beta_{1}, \beta_{2}, \ldots, \beta_{n}\right\|^{-1} \ldots\left\|\gamma_{1}, \gamma_{2}, \ldots, \gamma_{n}\right\|^{-1} \\
& \times\left(\sum\left\langle\alpha_{1}, \alpha_{i_{1}} \mid \alpha_{i_{2}}, \ldots, \alpha_{i_{n}}\right\rangle\right)\left(\sum\left\langle\beta_{1}, \beta_{i_{1}} \mid \beta_{i_{2}}, \ldots, \beta_{i_{n}}\right\rangle\right) \\
& \cdots\left(\sum\left\langle\gamma_{1}, \gamma_{i_{1}} \mid \gamma_{i_{2}}, \ldots, \gamma_{i_{n}}\right\rangle\right)
\end{aligned}
$$

such that $i_{1} \neq 1$ and $i_{1} \in\{1, \ldots, n\} \backslash\left\{i_{2}, \ldots, i_{n}\right\}$. By using Cauchy-Schwarz inequality we have

$$
\left|\left\langle\alpha_{1}, \alpha_{i_{1}} \mid \alpha_{i_{2}}, \ldots, \alpha_{i_{n}}\right\rangle\right| \leqslant\left\|\alpha_{1}, \ldots, \alpha_{n}\right\|\left|\beta_{1}, \ldots, \beta_{n}\left\|\cdots \mid \gamma_{1}, \ldots, \gamma_{n}\right\|=0 .\right.
$$

Also,

$$
\left|\left\langle\alpha_{1}, \alpha_{i_{1}} \mid \alpha_{i_{2}}, \ldots, \alpha_{i_{n}}\right\rangle\right|=\left|\left\langle\beta_{1}, \beta_{i_{1}} \mid \beta_{i_{2}}, \ldots, \beta_{i_{n}}\right\rangle\right|=\cdots=\left|\left\langle\gamma_{1}, \gamma_{i_{1}} \mid \gamma_{i_{2}}, \ldots, \gamma_{i_{n}}\right\rangle\right|=0,
$$

since, $\alpha_{1}$ should be equal one from $\alpha_{i_{2}}, \ldots, \alpha_{i_{n}}$, same case for $\beta_{1}, \ldots, \gamma_{1}$.

Then we get,

$$
\begin{aligned}
\left|\zeta\left(h_{1}, h_{2}, \ldots, h_{n}\right)\right| & =\left\|\alpha_{1}, \ldots, \alpha_{n}\right\|^{-1}\left\|\beta_{1}, \ldots, \beta_{n}\right\|^{-1} \cdots\left\|\gamma_{1}, \ldots, \gamma_{n}\right\|^{-1} \\
& \times\left\langle\alpha_{1}, \alpha_{1} \mid \alpha_{2}, \ldots, \alpha_{n}\right\rangle\left\langle\beta_{1}, \beta_{1} \mid \beta_{2}, \ldots, \beta_{n}\right\rangle \cdots\left\langle\gamma_{1}, \gamma_{1} \mid \gamma_{2}, \ldots, \gamma_{n}\right\rangle \\
& =\left\|\alpha_{1}, \ldots, \alpha_{n}\right\|\left\|\beta_{1}, \ldots, \beta_{n}\right\| \cdots\left\|\gamma_{1}, \ldots, \gamma_{n}\right\|
\end{aligned}
$$

such that $i_{1} \neq 1$ and $i_{1} \in\{1, \ldots, n\} \backslash\left\{i_{2}, \ldots, i_{n}\right\}$.

Then $\zeta$ is bounded of first index, such that

$$
\|\zeta\|_{1}=\left\|\alpha_{1}, \ldots, \alpha_{n}\right\|\left\|\beta_{1}, \ldots, \beta_{n}\right\| \cdots\left\|\gamma_{1}, \ldots, \gamma_{n}\right\|,
$$

condition (b) is hold. To prove (c) we will apply Hölder inequality on (3.6) to get

$$
\begin{aligned}
\left\|\zeta\left(h_{1}, h_{2}, \ldots, h_{n}\right)\right\| & \leqslant n^{\frac{n}{\sigma}}\left\|\alpha_{1}, \ldots, \alpha_{n}\right\|\left\|\beta_{1}, \ldots, \beta_{n}\right\| \cdots\left\|\gamma_{1}, \ldots, \gamma_{n}\right\| \\
& \times\left(\sum\left\|h_{1}, \alpha_{i_{2}}, \ldots, \alpha_{i_{n}}\right\|^{\tau}\right)^{\frac{1}{\tau}}\left(\sum\left\|h_{2}, \beta_{i_{2}}, \ldots, \beta_{i_{n}}\right\|^{\tau}\right)^{\frac{1}{\tau}} \\
& \cdots\left(\sum\left\|h_{n}, \gamma_{i_{2}}, \ldots, \gamma_{i_{n}}\right\|^{\tau}\right)^{\frac{1}{\tau}} .
\end{aligned}
$$

Take

$$
\begin{gathered}
h_{1}=n^{\frac{-1}{\tau}}\left\|\alpha_{1}, \ldots, \alpha_{n}\right\|^{-1}\left(\alpha_{1}+\cdots+\alpha_{n}\right) \\
h_{2}=n^{\frac{-1}{\tau}}\left\|\beta_{1}, \ldots, \beta_{n}\right\|^{-1}\left(\beta_{1}+\cdots+\beta_{n}\right) \\
\vdots \\
h_{n}=n^{\frac{-1}{\tau}}\left\|\gamma_{1}, \ldots, \gamma_{n}\right\|^{-1}\left(\gamma_{1}+\cdots+\gamma_{n}\right) .
\end{gathered}
$$


Therefore, by (2.1), we have

$$
\begin{aligned}
\left|\zeta\left(h_{1}, h_{2}, \ldots, h_{n}\right)\right| & =n^{\frac{-1}{\tau}}\left\|\alpha_{1}, \ldots, \alpha_{n}\right\|^{-1}\left(\sum\left\langle\alpha_{1}+\ldots+\alpha_{n}, \alpha_{i_{1}} \mid \alpha_{i_{2}}, \ldots, \alpha_{i_{n}}\right\rangle\right) \\
& \times n^{\frac{-1}{\tau}}\left\|\beta_{1}, \ldots, \beta_{n}\right\|^{-1}\left(\sum\left\langle\beta_{1}+\cdots+\beta_{n}, \alpha_{i_{1}} \mid \beta_{i_{2}}, \ldots, \beta_{i_{n}}\right\rangle\right) \\
& \ldots \\
& \times n^{\frac{-1}{\tau}}\left\|\gamma_{1}, \ldots, \gamma_{n}\right\|^{-1}\left(\sum\left\langle\gamma_{1}+\ldots+\gamma_{n}, \gamma_{i_{1}} \mid \gamma_{i_{2}}, \ldots, \gamma_{i_{n}}\right\rangle\right) \\
& =n^{\frac{-1}{\tau}}\left\|\alpha_{1}, \ldots, \alpha_{n}\right\|^{-1}\left(\sum\left\langle\alpha_{i_{1}}, \alpha_{i_{1}}, \mid \alpha_{i_{2}}, \ldots, \alpha_{i_{n}}\right\rangle\right) \\
& \times n^{\frac{-1}{\tau}}\left\|\beta_{1}, \ldots, \beta_{n}\right\|^{-1}\left(\sum\left\langle\beta_{i_{1}}, \beta_{i_{1}}, \mid \beta_{i_{2}}, \ldots, \beta_{i_{n}}\right\rangle\right) \\
& \ldots \\
& \times n^{\frac{-1}{\tau}}\left\|\gamma_{1}, \ldots, \gamma_{n}\right\|^{-1}\left(\sum\left\langle\gamma_{i_{1}}, \beta_{i_{1}}, \mid \gamma_{i_{2}}, \ldots, \gamma_{i_{n}}\right\rangle\right) \\
& =n^{\frac{-1}{\sigma}}\left\|\alpha_{1}, \ldots, \alpha_{n}\right\|^{-1} n\left\|\alpha_{1}, \ldots, \alpha_{n}\right\|^{2} \\
& \times n^{\frac{-1}{\tau}}\left\|\beta_{1}, \ldots, \beta_{n}\right\|^{-1} n\left\|\beta_{1}, \ldots, \beta_{n}\right\|^{2} \\
& \ldots \\
& \times n^{\frac{-1}{\tau}}\left\|\gamma_{1}, \ldots, \gamma_{n}\right\|^{-1} n\left\|\gamma_{1}, \ldots, \gamma_{n}\right\|^{2} \\
& =n^{\frac{-n}{\sigma}} n^{n}\left\|\alpha_{1}, \ldots, \alpha_{n}\right\|\left\|\beta_{1}, \ldots, \beta_{n}\right\| \ldots\left\|\gamma_{1}, \ldots, \gamma_{n}\right\| .
\end{aligned}
$$

This leads to

$$
\|\zeta\|_{\tau}=n^{\frac{n}{\tau}}\left\|\alpha_{1}, \ldots, \alpha_{n}\right\|\left\|\beta_{1}, \ldots, \beta_{n}\right\| \cdots\left\|\gamma_{1}, \ldots, \gamma_{n}\right\|,
$$

(c) has been proven.

Now, let $\zeta:\left(\mathrm{H}_{1} \times \mathrm{H}_{2} \times \cdots \times \mathrm{H}_{n}\right) \rightarrow \mathbb{R}$, be an $n$-linear functional defined on the $n$-inner product spaces

$$
\begin{aligned}
&\left(H_{1},\langle\cdot, \cdot \mid, \ldots,\rangle_{H_{1}}\right),\left(H_{2},\langle\cdot, \cdot \mid \cdot, \ldots, \cdot\rangle_{H_{2}}\right), \ldots,\left(H_{n},\langle\cdot, \cdot, \ldots, \cdot\rangle_{H_{n}}\right) \text { as } \\
& \zeta\left(h_{1}, h_{2}, \ldots, h_{n}\right)=\left(\sum\left\langle h_{1}, \alpha_{i_{1}} \mid \alpha_{i_{2}}, \ldots, \alpha_{i_{n}}\right\rangle_{H_{1}}\right)\left(\sum\left\langle h_{2}, \beta_{i_{1}} \mid \beta_{i_{2}}, \ldots, \beta_{i_{n}}\right\rangle_{H_{2}}\right) \\
&\left.\cdots\left(\sum\left\langle h_{n}, \gamma i_{1} \mid \gamma_{i_{2}}, \ldots, \gamma_{i_{n}}\right\rangle\right\rangle_{H_{n}}\right) \\
& \leqslant\left(\sum\left\|h_{1}, \alpha_{i_{2}}, \ldots, \alpha_{i_{n}}\right\|_{H_{1}}\right) \|\left(\sum\left\|\alpha_{i_{1}}, \alpha_{i_{2}}, \ldots, \alpha_{i_{n}}\right\|_{H_{1}}\right) \\
&\left(\sum\left\|h_{2}, \beta_{i_{2}}, \ldots, \beta_{i_{n}}\right\|_{H_{2}}\right) \|\left(\sum\left\|\beta_{i_{1}}, \beta_{i_{2}}, \ldots, \beta_{i_{n}}\right\|_{H_{2}}\right) \\
& \ldots \\
&\left(\sum\left\|h_{n}, \gamma_{i_{2}}, \ldots, \gamma_{i_{n}}\right\|_{H_{n}}\right) \|\left(\sum\left\|\gamma_{i_{1}}, \gamma_{i_{2}}, \ldots, \gamma_{i_{n}}\right\|_{H_{n}}\right) \\
& \leqslant\left(\sum\left\|h_{1}, \alpha_{i_{2}}, \ldots, \alpha_{i_{n}}\right\|_{H_{1}}\right) \|\left(\left\|\alpha_{i_{1}}, \alpha_{i_{2}}, \ldots, \alpha_{i_{n}}\right\|_{H_{1}}\right) \\
&\left(\sum\left\|h_{2}, \beta_{i_{2}}, \ldots, \beta_{i_{n}}\right\|_{H_{2}}\right) \|\left(\left\|\beta_{i_{1}}, \beta_{i_{2}}, \ldots, \beta_{i_{n}}\right\|_{H_{2}}\right) \\
& \ldots \\
&\left(\sum\left\|h_{n}, \gamma_{i_{2}}, \ldots, \gamma_{i_{n}}\right\|_{H_{n}}\right) \|\left(\left\|\gamma_{i_{1}}, \gamma_{i_{2}}, \ldots, \gamma_{i_{n}}\right\|_{H_{n}}\right) .
\end{aligned}
$$


Now, take

$$
h_{1}=\frac{\alpha_{1}}{\left\|\alpha_{1}, \alpha_{2}, \ldots, \alpha_{n}\right\|}, h_{2}=\frac{\beta_{1}}{\left\|\beta_{1}, \beta_{2}, \ldots, \beta_{n}\right\|}, \ldots, h_{n}=\frac{\gamma_{1}}{\left\|\gamma_{1}, \gamma_{2}, \ldots, \gamma_{n}\right\|} .
$$

Then we get

$$
\begin{aligned}
&\left(\sum\left\|h_{1}, \alpha_{i_{2}}, \ldots, \alpha_{i_{n}}\right\|_{H_{1}}\right) \leqslant 1,\left(\sum\left\|h_{2}, \beta_{i_{2}}, \ldots, \beta_{i_{n}}\right\|_{H_{2}}\right) \leqslant 1, \\
& \ldots,\left(\sum\left\|h_{n}, \gamma_{i_{2}}, \ldots, \gamma_{i_{n}}\right\|_{H_{n}}\right) \| \leqslant 1 .
\end{aligned}
$$

From, (3.19) and (3.20) we get (3.9), The same proof of method (d) can be used to find ( 3.10) with helping (c). To prove (f), suppose that (3.9) is satisfying, it implies that each term of the total is under 1 , as outcome

$$
\left(\sum\left\|h_{1}, \alpha_{i_{2}}, \ldots, \alpha_{i_{n}}\right\|^{\tau}\right)\left(\sum\left\|h_{2}, \beta_{i_{2}}, \ldots, \beta_{i_{n}}\right\|^{\tau}\right) \cdots\left(\sum\left\|h_{n}, \gamma_{i_{2}}, \ldots, \gamma_{i_{n}}\right\|^{\tau}\right) \leqslant 1 .
$$

Then

$$
\|\zeta\|_{1} \leqslant\|\zeta\|_{\tau}
$$

In the opposite direction, let (3.10) is hold, therefore

$$
\left(\sum\left\|h_{1}, \alpha_{i_{2}}, \ldots, \alpha_{i_{n}}\right\|^{\tau}\right)\left(\sum\left\|h_{2}, \beta_{i_{2}}, \ldots, \beta_{i_{n}}\right\|^{\tau}\right) \cdots\left(\sum\left\|h_{n}, \gamma_{i_{2}}, \ldots, \gamma_{i_{n}}\right\|^{\tau}\right) \leqslant 1,
$$

this leads to

$$
\left(\sum\left\|h_{1}, \alpha_{i_{2}}, \ldots, \alpha_{i_{n}}\right\|\right)\left(\sum\left\|h_{2}, \beta_{i_{2}}, \ldots, \beta_{i_{n}}\right\|\right) \cdots\left(\sum\left\|h_{n}, \gamma_{i_{2}}, \ldots, \gamma_{i_{n}}\right\|\right) \leqslant n^{\frac{n}{\sigma}},
$$

and

$$
\begin{aligned}
\left(\sum\left\|\left(\frac{1}{n} h_{1}^{\frac{\sigma}{n}}\right)^{\frac{n}{\sigma}}, \alpha_{i_{2}}, \ldots, \alpha_{i_{n}}\right\|\right) & \left(\sum\left\|\left(\frac{1}{n} h_{2}^{\frac{\sigma}{n}}\right)^{\frac{n}{\sigma}}, \beta_{i_{2}}, \ldots, \beta_{i_{n}}\right\|\right) \\
& \cdots\left(\sum\left\|\left(\frac{1}{n} h_{n}^{\frac{\sigma}{n}}\right)^{\frac{n}{\sigma}}, \gamma_{i_{2}}, \ldots, \gamma_{i_{n}}\right\|\right) \leqslant 1 .
\end{aligned}
$$

Then we obtain

$$
\left\|\zeta\left(\frac{h_{1}}{n^{\frac{1}{\sigma}}}, \frac{h_{2}}{n^{\frac{1}{\sigma}}}, \ldots, \frac{h_{n}}{n^{\frac{1}{\sigma}}},\right)\right\|_{\tau} \leqslant\left\|\zeta\left(h_{1}, h_{2}, \ldots, h_{n}\right)\right\|_{1} .
$$

Hence,

$$
\left\|\zeta\left(h_{1}, h_{2}, \ldots, h_{n}\right)\right\|_{\tau} \leqslant n^{\frac{n}{\sigma}}\left\|\zeta\left(h_{1}, h_{2}, \ldots, h_{n}\right)\right\|_{1} .
$$

We conclude that $\left\|\zeta\left(h_{1}, h_{2}, \ldots, h_{n}\right)\right\|_{1},\left\|\zeta\left(h_{1}, h_{2}, \ldots, h_{n}\right)\right\|_{\tau}$ are equivalent by

$$
\left\|\zeta\left(h_{1}, h_{2}, \ldots, h_{n}\right)\right\|_{1} \leqslant\left\|\zeta\left(h_{1}, h_{2}, \ldots, h_{n}\right)\right\|_{\tau} \leqslant n^{\frac{n}{\sigma}}\left\|\zeta\left(h_{1}, h_{2}, \ldots, h_{n}\right)\right\|_{1} .
$$


From all of the above, it is possible to extract the result of existence and uniqueness of the approximate solution set to the $n$-linear functionals $\mathfrak{I}$ space useing nonexpansive mapping from type $(\mu, \sigma)$. We assume that $\phi \neq \mathfrak{I} \subset \mathrm{n}$-Banach sapces then we draw the next results

Definition 3.13. Let $\mathfrak{I} \neq \phi$ be an $n$-Banach space, we say $\rho: \mathfrak{I} \rightarrow H$ is $(\mu, \sigma)$-nonexpansive mappings, if for all $h_{i}, s_{i}, \hbar_{2}, \ldots, \hbar_{n} \in H, \zeta\left(h_{i}\right), \varphi\left(s_{i}\right) \subset \mathfrak{I} \in H$ and $, \mu, \sigma \in$ $\mathbb{R}, i \in(1, \ldots, n)$ we have

$$
\begin{aligned}
& \left\|\rho \zeta\left(h_{i}\right)-\rho \varphi\left(s_{i}\right), \hbar_{2}, \ldots, \hbar_{n}\right\|^{2} \\
& \leqslant \mu\left\|\rho \zeta\left(h_{i}\right)-\varphi\left(s_{i}\right), \hbar_{2}, \ldots, \hbar_{n}\right\|^{2}+\mu\left\|\rho \varphi\left(s_{i}\right)-\zeta\left(h_{i}\right), \hbar_{2}, \ldots, \hbar_{n}\right\|^{2} \\
& +\sigma\left\|\rho \zeta\left(h_{i}\right)-\zeta\left(h_{i}\right), \hbar_{2}, \ldots, \hbar_{n}\right\|^{2}+\sigma\left\|\rho \varphi\left(s_{i}\right)-\varphi\left(s_{i}\right), \hbar_{2}, \ldots, \hbar_{n}\right\|^{2} \\
& +2(\mu-\sigma)\left\|\zeta\left(h_{i}\right)-\varphi\left(s_{i}\right), \hbar_{2}, \ldots, \hbar_{n}\right\|^{2} .
\end{aligned}
$$

Remark 3.14. Suppose that, the equivalence relationship between bounded n-linear functionals can be transformed in some case into something of a congruence, such that the relationship of (3.11) will be transformed into $\zeta\left(h_{i}\right)=\varphi\left(s_{i}\right)$, this means the special case affects into Definition 3.13 we get

$$
\left\|\zeta\left(h_{i}\right), \hbar_{2}, \ldots, \hbar_{n}\right\| \leqslant\left\|\rho \zeta\left(h_{i}\right), \hbar_{2}, \ldots, \hbar_{n}\right\| .
$$

Definition 3.15. Let $\zeta\left(h_{i}\right), \varphi\left(s_{i}\right) \subset \mathfrak{I} \in H$, a point $\left(\left\|\zeta\left(h_{1}\right), h_{2}, \ldots, h_{n}\right\| \rightarrow \mathbb{R}\right) \in \mathfrak{I}$ is called a fixed point for $\rho$ when $\left\|\rho \zeta\left(h_{1}\right), h_{2}, \ldots, h_{n}\right\|=\left\|\zeta\left(h_{1}\right), h_{2}, \ldots, h_{n}\right\|$. A set $\mathfrak{I} \supset$ $\left\|\zeta\left(h_{i}\right), h_{2}, \ldots, h_{n}\right\| \rightarrow \mathbb{R}$ is called an approximate fixed points for $\rho$ when

$$
\left\|\rho \zeta\left(h_{i}\right), h_{2}, \ldots, h_{n}\right\|=\left\|\zeta\left(h_{i}\right), h_{2}, \ldots, h_{n}\right\|,
$$

for all $h_{2}, \ldots, h_{n} \in H$, and $i_{1} \in(1, \ldots, n) \backslash\left(i_{2}, \ldots, i_{n}\right)$.

Theorem 3.16. Let $\rho: \mathfrak{I} \rightarrow \mathrm{H}$ be $(\mu, \sigma)$-non-expansive mappings. Then $\rho\left(\zeta\left(h_{i}\right)\right)=\zeta\left(h_{i}\right)$ for all $\zeta\left(h_{i}\right) \in H, i \in(1, \ldots, n)$.

Proof. First: Existence

Let,

$$
\rho\left(\zeta\left(h_{1}, h_{2}, \ldots, h_{n}\right)\right)=\sum_{i_{1}, i_{2}, \ldots, i_{n} \in\{1,2, \ldots, n\}} \zeta\left(h_{i_{1}}, h_{i_{2}}, \ldots, h_{i_{n}}\right) \rightarrow \mathbb{R}
$$

For all $h_{1}, h_{2}, \ldots, h_{n} \in H$. Then, for all $n$-linear functionals $\zeta, \varphi \subset \mathfrak{I} \in H$ and $h_{i_{1}}, \ldots, h_{i_{n}} \in h_{1}, \ldots, h_{n}, s_{i_{1}}, \ldots s_{i_{n}} \in s_{1}, \ldots s_{n}$, where $\left\{x_{i_{1}}, x_{i_{2}}, \ldots, x_{i_{n}}\right\}$, are linear independent subsets of $H$, such that $x$ it takes the following values sequentially, $\alpha, \beta, \gamma, a, b, c, \hat{\alpha}$. 
We have

$$
\begin{aligned}
\left\langle\rho \zeta\left(h_{i}\right)-\rho \varphi\left(s_{i}\right)\right\rangle= & \sum\left\langle h_{i_{1}}, \alpha_{i_{1}} \mid \alpha_{i_{2}}, \ldots, \alpha_{i_{n}}\right\rangle \sum\left\langle h_{i_{2}}, \beta_{i_{1}} \mid \beta i_{2}, \ldots, \beta_{i_{n}}\right\rangle \\
& \ldots \sum\left\langle h_{i_{n}}, \gamma_{i_{1}} \mid \gamma_{i_{2}}, \ldots, \gamma_{i_{n}}\right\rangle \\
& -\sum\left\langle s_{i_{1}}, a_{i_{1}} \mid a_{i_{2}}, \ldots, a_{i_{n}}\right\rangle \sum\left\langle s_{i_{2}}, b_{i_{1}} \mid b i_{2}, \ldots, b_{i_{n}}\right\rangle \\
& \ldots \sum\left\langle s_{i_{n}}, c_{i_{1}} \mid c_{i_{2}}, \ldots, c_{i_{n}}\right\rangle, \\
= & \sum\left\langle h_{i_{1}}-s_{i_{1}}, \alpha_{i_{1}}-a_{i_{1}} \mid\left(\alpha_{i_{2}}, \ldots, \alpha_{i_{n}}-a_{i_{2}}, \ldots, a_{i_{n}}\right)\right\rangle \\
& \sum \sum\left\langle h_{i_{2}}-s_{i_{2}}, \beta_{i_{1}}-b_{i_{1}} \mid\left(\beta i_{2}, \ldots, \beta_{i_{n}}-b i_{2}, \ldots, b_{i_{n}}\right)\right\rangle \\
& \ldots \sum\left\langle h_{i_{n}}-s_{i_{n}}, \gamma_{i_{1}}-c_{i_{i}} \mid\left(\gamma_{i_{2}}, \ldots, \gamma_{i_{n}}-c_{i_{2}}, \ldots, c_{i_{n}}\right)\right\rangle \\
& \leqslant\left\|h_{i_{1}}-s_{i_{1}}, \hat{\alpha}_{i_{2}}, \ldots, \hat{\alpha}_{i_{n}}\right\|\left\|h_{i_{2}}-s_{i_{2}}, \hat{\beta}_{i_{2}}, \ldots, \hat{\beta}_{i_{n}}\right\| \\
& \ldots\left\|h_{i_{n}}-s_{i_{n}}, \hat{\gamma}_{i_{2}}, \ldots, \hat{\gamma}_{i_{n}}\right\|, \\
& \leqslant\left\|\hat{\alpha}_{i_{2}}, \ldots, \hat{\alpha}_{i_{n}}\right\|\left\|\hat{\beta}_{i_{2}}, \ldots, \hat{\beta}_{i_{n}}\right\|\left\|\hat{\gamma}_{i_{2}}, \ldots, \hat{\gamma}_{i_{n}}\right\| \\
& \cdots\left\|h_{i_{n}}-s_{i_{n}}, h_{i_{1}}-s_{i_{1}}, \ldots, h_{i_{n}}-s_{i_{n}}\right\| .
\end{aligned}
$$

We get

$$
\left\|\rho \zeta\left(h_{i}\right)-\rho \zeta\left(s_{i}\right)\right\|^{2} \leqslant\left\|\hat{\alpha}_{i_{2}}, \ldots, \hat{\alpha}_{i_{n}}\right\|\left\|\hat{\beta}_{i_{2}}, \ldots, \hat{\beta}_{i_{n}}\right\|\left\|\hat{\gamma}_{i_{2}}, \ldots, \hat{\gamma}_{i_{n}}\right\| \cdots\|\| \zeta(h)-\varphi(s) \|^{2} .
$$

Hence,

$$
\left\|\rho \zeta\left(h_{i}\right)-\rho \zeta\left(s_{i}\right)\right\|^{2} \leqslant \sigma\left\|\rho \zeta\left(h_{i}\right)-\zeta\left(h_{i}\right)\right\|^{2}+\sigma\left\|\rho \varphi\left(s_{i}\right)-\varphi\left(s_{i}\right)\right\|^{2}-2 \sigma\left\|\zeta\left(h_{i}\right)-\varphi\left(s_{i}\right)\right\|^{2} .
$$

Where,

$$
\begin{array}{r}
\sigma\left\|\rho \zeta\left(h_{i}\right)-\zeta\left(h_{i}\right)\right\|^{2}+\sigma\left\|\rho \varphi\left(s_{i}\right)-\varphi\left(s_{i}\right)\right\|^{2}-2 \sigma= \\
\left\|\hat{\alpha}_{i_{2}}, \ldots, \hat{\alpha}_{i_{n}}\right\|\left\|\hat{\beta}_{i_{2}}, \ldots, \hat{\beta}_{i_{n}}\right\|\left\|\hat{\gamma}_{i_{2}}, \ldots, \hat{\gamma}_{i_{n}}\right\|
\end{array}
$$

Then $\rho$ has approximate fixed points.

Second: uniqueness

(3.11) in Theorem 3.12, told us that all n-linear functional spaces are equivalent in any bounded, hence they have a same solution which means same approximate fixed points.

\section{Conclusion}

In this paper, we have successfully established the approximate fixed points set for $n$ linear functionals spaces $\mathfrak{I}$ such that the class of $(\mu, \sigma)$-nonexpansive mappings are equivalent, where $\mathfrak{I}$ is a non-empty bounded subset of an $n$-Banach space $\mathrm{H}$. 


\section{Acknowledgements}

We would like to thank the referees very much for their valuable comments and suggestions.

\section{References}

[1] Gahler S (1969). Untersuchungen uber verallgemeinertem-metrische raume. Math. Nachr. 40(I): 16 -189.

[2] Gahler S (1969). Untersuchungen uber verallgemeinertem-metrische raume. Math. Nachr. 40(II): 229264.

[3] Gahler S (1969). Untersuchungen uber verallgemeinertem-metrische raume. Math. Nachr. 40(III): 23-36.

[4] Misiak A (1989). n-Inner product spaces. Mathematische Nachrichten 140(1): 299-319. https://doi.org/10.1002/mana.19891400121

[5] Batkunde $\mathrm{H}$ and Gunawan $\mathrm{H}$ (2018). On the topology of n-normed spaces with respect to norms of its quotient spaces. arXiv:1810.07975. https://arxiv.org/abs/1810.07975

[6] Batkunde H, Gunawan $\mathrm{H}$ and Neswan O (2018). n-Normed spaces with norms of its quotient spaces. J. Phys. Conf. Series 1097: 012079. https://doi:10.1088/1742-6596/1097/1/012079

[7] Batkunde H, Gunawan H and Neswan O (2019). A revisit to $n$-Normed spaces through its quotient spaces. arXiv preprint, arXiv:1904.00593. https://arxiv.org/abs/1904.00593

[8] Eghbali N, Rassias J and Taheri M (2016). On the Stability of ak-Cubic Functional Equation in Intuitionistic Fuzzy n-Normed Spaces. Results in Math. 70:233-248. https://doi.org/10.1007/s00025-015-0476-9

[9] Gunawan H (2001). On n-normed spaces. Int. J. Math. Math. Sci. 27(10): 631-639.

[10] Huang X and Tan A (2018). Mappings of preserving n-distance one in n-normed spaces. Aequationes Math. 92(3): 401-413. https://doi.org/10.1007/s00010-018-0539-6

[11] Ekariani S, Gunawan H and Idris M (2013). contractive mapping theorem on then-normed space of psummable sequences. J. Math. Anal. 4(1): 1-7.

[12] Gunawan H (2001). The space of p-summable sequences and its natural n-norm. Bull.Austral. Math. Soc. 64(1): 137-147. https://doi.org/10.1017/S0004972700019754

[13] Srivastava J and Singh P (2016). On some Cauchy sequences defined in (lp) considered as n-normed space. J. Rajasthan Acad. Phys. Sci. 15(1-2): 107-116.

[14] Meitei S and Singh M (2018). On bounded n-linear operators. J. Math. Comput. Sci. 8(2): 196-215. https://doi.org/10.28919/jmcs/3446

[15] Gunawan H, Sukaesih E and Neswan O (2015). Fixed point theorems on bounded sets in an n-normed space. J. Math. Anal. 3: 51-58.

[16] Banach S (1922). Sur les opérations dans les ensembles abstraits et leur application aux équations intégrales. Fund. Math. 3(1): 133-181.

[17] Amini-Harandi A, Fakher M and Hajisharifi H (2018). The fixed point of $\alpha$-nonexpansive mappings. J. Math. Anal. Appl. 467(2): 1168-1173. https://doi.org/10.1016/j.jmaa.2018.07.058

[18] Amini-Harandi A, Fakhar M and Hajisharifi H (2016). Weak fixed point property for nonexpansive mappings with respect to orbits in Banach spaces. J. Fixed Point Theory Appl. 18(3): 601-607. https://doi.org/10.1007/s11784-016-0310-3

[19] Kirk W and Shahzad N (2018). Normal structure and orbital fixed point conditions. J. Math. Anal. Appl. 463(2): 461-476. https://doi.org/10.1016/j.jmaa.2018.02.022

[20] Xianbing W (2020). Fixed Point Problems for Nonexpansive Mappings in Bounded sets of Banach Spaces. Adv. Math. Phys. 2020, Article ID 9182016: 6. https://doi.org/10.1155/2020/9182016

[21] Afshari H, Abdo MS, Alzabut J (2020). Further results on existence of positive solutions of generalized fractional boundary value problems. Adv. Differ. Equ. 2020(1):600. https://doi.org/10.1186/s13662020-03065-2

[22] Abdeljawad T, Karapınar E and Taş K (2011). Existence and uniqueness of a common fixed point on partial metric spaces. Appl. Math. Lett. 24(11): 1900-1904. https://doi.org/10.1016/j.aml.2011.05.014

[23] Mlaiki N, Aydi H, Souayah N and Abdeljawad T (2018). Controlled metric type spaces and the related contraction principle. Mathematics 6(10): 194. https://doi.org/10.3390/math6100194

[24] Abdeljawad T, Karapınar E and Taş K (2012). A generalized contraction principle with control functions on partial metric spaces. Computers \& Mathematics with Applications 63(3): 716-719. https://doi.org/10.1016/j.camwa.2011.11.035 
[25] Abdeljawad T, Mlaiki N, Aydi H and Souayah N (2018). Double controlled metric type spaces and some fixed point results. Mathematics 6(12): 320. https://doi.org/10.3390/math6120320

[26] Panda SK, Abdeljawad T and Ravichandran C (2020). Novel fixed point approach to AtanganaBaleanu fractional and Lp-Fredholm integral equations. Alexandria Eng. J. 95(4): 1959-1970. https://doi.org/10.1016/j.aej.2019.12.027

[27] Brzdek J and Cieplinski K (2019). A fixed point theorem in n-Banach spaces and Ulam stability. J. Math. Anal. Appl. 470(1): 632-646. https://doi.org/10.1016/j.jmaa.2018.10.028

[28] Batkunde H, Gunawan H and Pangalel Y (2013). Bounded linear functionals on the n-normed spaces of p-summable sequence. Acta Univ. M. Belii. Ser. Math. 21: 71-80.

[29] Kristiantoo T, Wibawa T and Gunawan H (2013). Equivalence relation of n-norms on a vector space. Mat. Vesnik. 65(4): 488-493.

[30] Gantmacher F (2000). The Theory of Matrices. AMS Chelsea Publishing, 1: 252-253. 